\title{
PREDICTING CONSTRUCTION PROJECT DURATION WITH SUPPORT VECTOR MACHINE
}

\author{
Silvana Petruseva ${ }^{1}$, Valentina Zileska-Pancovska ${ }^{2}$, Vahida Zujo ${ }^{3}$ \\ ${ }^{I}$ Asist.Prof., Mathematics Department, Faculty of Civil Engineering, Macedonia, silvana@gf.ukim.edu.mk \\ ${ }^{2}$ Full Prof., Department of Management, Faculty of Civil Engineering, Macedonia, valentinazp@gf.ukim.edu.mk \\ ${ }^{3}$ Prof., Department of Management, Faculty of Civil Engineering, Boznia and Herzegoovina, vahida.zujo@unmo.ba
}

\begin{abstract}
This paper presents a forecasting model for construction time, using support vector machine (SVM) - recently one of the most accurate predictive models.

Every construction contract contains project deadline as an essential element of the contract. The practice shows that a considerably present problem is that of non-compliance of the contracted and real construction time. It's often the case that construction time is determined arbitrarily. The produced dynamic plans are only of formal character and not a reflection of the real possibilities of the contractor.
\end{abstract}

First, a linear regression model has been applied to the data for 75 objects, using Bromilow's "time cost" model. After that a support vector machine model to the same data was applied and significant improvement of the accuracy of the prediction was obtained.

Keywords construction time, construction costs, artificial neural network, linear regression, support vector machine

\section{INTRODUCTION}

Key data on the total of 75 buildings constructed in the Federation of Bosnia and Herzegovina have been collected through field studies. Chief engineers of construction companies have been interviewed on contractual and actually incurred costs and terms. The collected data contain information for the contracted and real time of construction, the contracted and real price of construction and there are also data for the use of these 75 objects and for the year of construction .

Australian Bromilow was the first who investigated the relation between construction price and construction time. From his research stems the well known "time - cost" model [3] Afterward the model has been confirmed in many countries worldwide: UK, China, Malaysia, Croatia etc. [15], [5],[8],[4].

In Bosnia and Herzegovina similar research was first conducted in 2008 [38]. The research included seven groups of objects and "time - cost" model was established for all seven groups.

This paper will present the research results conducted on 75 structures constructed in the period from 1999 to 2011, in the field of civil engineering and building construction, as well as hydro construction
Data were collected for a total of 75 structures built in the Federation of Bosnia and Herzegovina in the period from 1999 to 2011 using questionnaires and interviews with contractors. Key data are:

- Object Type;

- Construction Year;

- Contracted And Realized Construction Time;

- Contracted And Realized Construction Price;

- Reasons For Non-Compliance Of Deadline.

From a total of 75 structures, disregard of the contracted deadline was registered at 55 of them $(73 \%)$, disregard of the contracted price was registered at 40 structures (53\%), while simultaneously the contracted deadline and the contracted price overrun were registered at 36 structures (48\%). Maximum contracted deadline overrun was $100 \%$, and price $68.75 \%$ while the average contracted deadline has been exceeded for $11.55 \%$ and $2.77 \%$ for the price. Contracted deadline reduction was registered at 11 structures $(14.67 \%)$, while simultaneously the contracted deadline and the contracted price reduction was registered at 2 structures $(2.67 \%)$ [39].

Total of five different reasons for non-compliance appear:

- approvals and permits;

- climate;

- incomplete and inaccurate technical documentation; 
- material delivery;

- Terms of financing.

This paper presents comparison of two predictive models for construction time. We shall first present linear regression model which has been applied to these data for predicting of the construction time, and after that support vector machine (SVM) predictive model applied to the same data. Short overview of linear regression and SVM predictive model will be presented also.

\section{LINEAR REGRESSION PREDICTION MODEL FOR CONSTRUCTION PROJECT DURATION}

Linear regression is an approach to model the relationship between a scalar dependant variable $\mathbf{Y}$ and one or more explanatory variables denoted $\mathbf{x}$. The case of one explanatory variable is called simple linear regression. For more than one explanatory variable, it is called multiple linear regression [35].

In linear regression data are modeled using linear predictor functions and unknown model parameters are estimated from the data.

A linear regression model fits a linear function to a set of data points. The form of the function is:

$$
\boldsymbol{Y}=\beta_{0}+\beta_{1} x_{1}+\beta_{2} x_{2}+\ldots \ldots+\beta_{n} x_{n}
$$

Where $Y$ is the target variable, $\boldsymbol{x}_{1}, \boldsymbol{x}_{2}, \ldots \boldsymbol{x}_{n}$, are the predictor variables, and $\beta_{1}, \beta_{2}, \ldots \ldots \beta_{n}$ are coefficients that multiply the predictor variables. $\beta_{0}$ is a constant.

Since linear regression is restricted to fitting linear (straight line/plane) functions to data, it rarely works as well on realworld data as more general techniques such as neural networks which can model non-linear functions. However, linear regression has many practical uses and a number of strengths. For example, linear regression analysis can be applied to quantify the strength of the relationship between $\mathbf{Y}$ and the $\mathbf{x}_{j}$, to assess which $\mathbf{x}_{j}$ may have no relationship with $\mathrm{Y}$ at all, and to identify which subsets of the $\mathbf{x}_{j}$ contain redundant information about $\mathrm{Y}$, also linear regression models are simple and require minimum memory to implement, so they work well on embedded controllers that have limited memory space [24].

For the prediction of the construction time we shall use Bromilow's "time cost"model given in Eq. (1).

$$
T=K \cdot C^{B}
$$

Where: $T$ - contracted time; $C$ - contracted price; $K$ - Model parameter that is a specific way to measure productivity because it shows the average time needed for the construction of a monetary value;

$B$ - Model parameters that show time dependence of costs change.

For the requirements of linear regression model, we shall write this model in linear form, using logarithmic transformation [4], as shown in Eq. (2):

$$
\ln \mathrm{T}=\ln \mathrm{K}+\mathrm{B} \ln \mathrm{C}
$$

The linear form of the equation allows usage of the simple statistical procedure, i.e., a single linear regression. We shall determine the values of parameters $\mathrm{K}$ and $\mathrm{B}$ in this model. For creating the linear regression model for predicting of the construction time for the 75 objects, DTREG software package was used [25].

The results of the DTREG analysis of the model are presented in Table 1 to Table 3 bellow.

Considering Eq. (2), variable $\ln$ (real time) is used as target variable, and $\ln ($ real price) as predicted variable.

From the Table 1 we can read the coefficients of the linear regression model, and they are: $\mathbf{B}=\mathbf{0 . 5 5 0 2 0 8}$ (which is multiplied with variable $\ln ($ real price), and the constant $\mathbf{l n K}=$ 2.37546 and from here $K=\mathrm{e}^{-2.37546}$.

The model will be: $\quad \mathbf{T}=\mathbf{e}^{-2.37546} \mathbf{C}^{\mathbf{0 . 5 5 0 2 0 8}}$

Part of the data are used for training of the model (training data, Table 2), and part of the data are used for validation of the model (Table 3).

We shall estimate the accuracy of the model from the statistics of validation data (Table 3):

Most often used estimators of a model are $\mathbf{R}^{\mathbf{2}}$ and MAPE. In statistics, the coefficient of determination denoted $\mathbf{R}^{\mathbf{2}}$ indicates how well data points fit a line or curve; it is a measure of global fit of the model. In linear regression $\mathbf{R}^{\mathbf{2}}$ equals the square of Pearson correlation coefficient between observed and modeled (predicted) data values of the dependant variable. $\mathbf{R}^{2}$ is an element of $[0,1]$ and is often interpreted as the proportion of the response variation "explained" by the regressors in the model. So, the value $\mathbf{R}^{\mathbf{2}}=\mathbf{0 . 7 3 3 4 1}$ from our model may be interpreted: around $73 \%$ of the variation in the response can be explained by the explanatory variables. The remaining $27 \%$ can be attributed to unknown, lurking variables or inherent variability [36].

MAPE (mean absolute percentage error) is a measure of accuracy of a method for constructing fitted times series 
values in statistics. It usually expresses accuracy as a means that the error of the model is around $\mathbf{1 0 \%}$. percentage [37]. For this model MAPE= 10.355481, which

Table - 1: Linear regression model for predicting real time of construction which depends on real price of construction

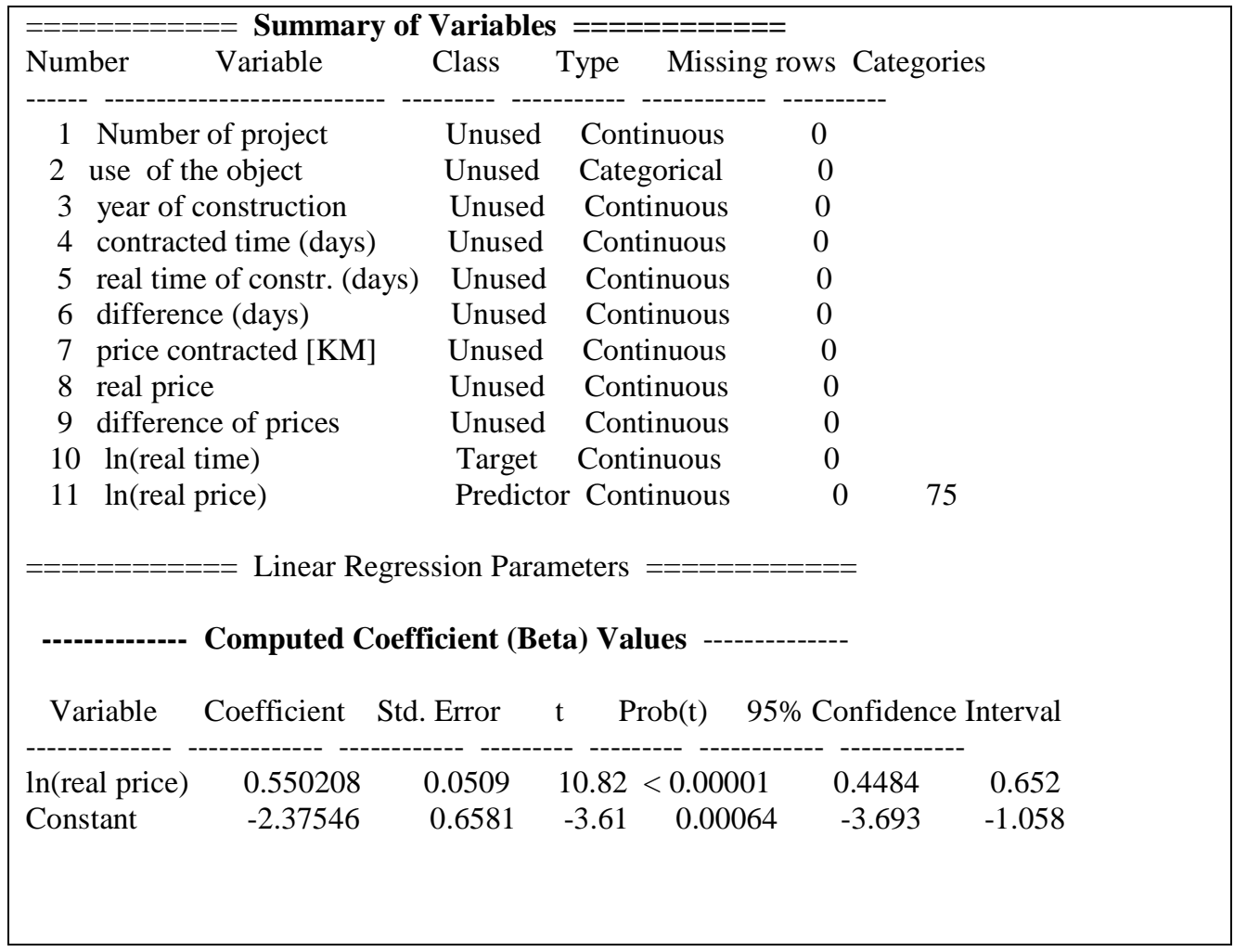


Table-2: Statistics for training data for the linear regression model

--- Training Data ---
Mean target value for input data $=4.7038902$
Mean target value for predicted values $=4.7038902$
Variance in input data $=0.8306976$
Residual (unexplained) variance after model fit $=$
0.2753121
Proportion of variance explained by model $\left(\mathrm{R}^{\wedge} 2\right)=$
$0.66858(66.858 \%)$
Coefficient of variation $(\mathrm{CV})=0.111546$
Normalized mean square error $(\mathrm{NMSE})=0.331423$
Correlation between actual and predicted $=0.817666$
Maximum error $=1.7033044$
RMSE (Root Mean Squared Error) $=0.5247019$
MSE (Mean Squared Error) $=0.2753121$
MAE (Mean Absolute Error) $=0.4108599$
MAPE (Mean Absolute Percentage Error) $=9.2084657$

\section{PREDICTING WITH SUPPORT VECTOR MACHINES (SVM) MODEL}

\subsection{Support Vector Machines}

In recent years, machine learning has become a focal point in artificial intelligence. Support vector machines are a relatively new, rapidly developing field of research - general formulation for learning machines. SVMs perform exceptionally well on pattern classification, function approximation, and regression problems.

SVM is a class of learning algorithms which has been proved in recent years to be superior to the conventional Neural Network method for both classification and regression tasks.

Support vector machine (SVM) has been first introduced by Vapnik. There are two main categories for support vector machines: support vector classification (SVC) and support vector regression (SVR). SVM is a learning system using a high dimensional feature space. It yields prediction functions that are expanded on a subset of support vectors. A version of a SVM for regression has been proposed in 1997 by Vapnik, Steven Golowich, and Alex Smola [33]. This method is called support vector regression (SVR).

The SV algorithm is a nonlinear generalization of the Generalized Portrait algorithm developed in Russia in the sixties [28], [29]. As such, it is firmly grounded in the
Table-3: Statistics for validation data used for estimation of the accuracy of the model framework of statistical learning theory, or VC theory, which has been developed over the last three decades by Vapnik and Chervonenkis [30], Vapnik [31],[32]. In a nutshell, VC theory characterizes properties of learning machines which enable them to generalize well to unseen data.

In its present form, the SV machine was largely developed at AT\&T Bell Laboratories by Vapnik and co-workers [2], [11], [9],[20], [21], [33]. Due to this industrial context, SV research has up to date had a sound orientation towards real-world applications.

In regression and time series prediction applications, excellent performances were obtained [18], [10],[27],[17]. A snapshot of the state of the art in SV learning was recently taken at the annual Neural Information Processing Systems conference [22]. SV learning has now evolved into an active area of research. Moreover, it is in the process of entering the standard methods toolbox of machine learning [12],[7], [14]. [23] by Sch"olkopf and Smola contains a more in-depth overview of SVM regression.

SVM's are new training method which is developed in the frame of structural risk minimization (SRM). SVM belongs to the group of supervised learning methods. They also belong to the group of nonparametric models, i.e. models for which parameters are not defined in advance, but their number depends on data for training, so that the ability of the model to interpolate data depends on the complexity of data. 
Figure 1 shows general model of SVM which is identical to the general model of multilayer preceptron neural network (MLP NN). The difference is in the way of training. Connections in the output layer are the weights $\mathbf{w}_{\mathbf{i}}$ and their meaning is identical as at NN after the training [16].

For all feed-forward NNs there is an ability of using support vector learning (SVL) algorithms in the process of learning. So, while the back-propagation algorithm is for training multilayer perceptron, SVL algorithm has more generic nature, and because of that has broad applicability [13]. Data for training consist of two sets of variables: 1) input (predictor) variables $\mathbf{x}_{\mathbf{i}}$, which are being chosen arbitrary from the input set $X$, and 2) response from the system $\mathbf{y}_{\mathbf{i}}$ (target variable) which belongs to the output set of data Y. In the parlance of SVM literature, a predictor variable is called an attribute, and a transformed attribute that is used to define the hyperplane is called a feature.

The task of choosing the most suitable representation is known as feature selection. A set of features that describes one case (i.e., a row of predictor values) is called a vector.

SVM belongs to kernel methods, i.e. methods with which nonlinear learning problem is transformed in linear using kernel functions. This is accomplished by mapping of the input space into output multidimensional space. The solving of the problem, whether it is a problem of classification or regression, will be accomplished in the new multidimensio-nal space.

Figure 2 shows the problem of classification of data which in input space is nonlinear problem. By mapping in the new multidimensional space, the ability of linear separation is given.

An SVM analysis finds the line (or, in general, hyperplane) that is oriented so that the margin between the support vectors is maximized. In the figure bellow (Fig.3), the line in the right panel is superior to the line in the left panel.

If all analyses consisted of two-category target variables with two predictor variables, and the cluster of points could be divided by a straight line, solving the problem would be easy. Unfortunately, this is not generally the case, so SVM must deal with (a) more than two predictor variables, (b) separating the points with non-linear curves, (c) handling the cases where clusters cannot be completely separated, and (d) handling classifications with more than two categories

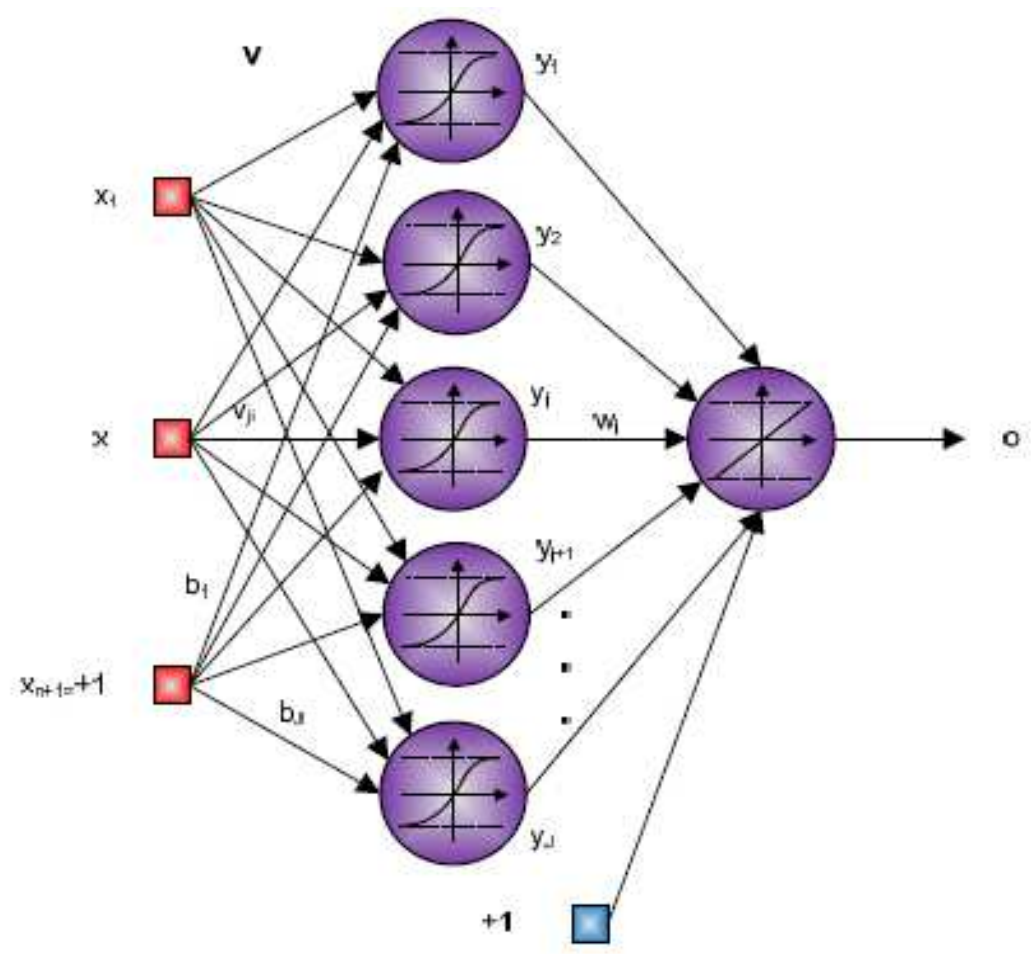

Fig-1: General model of SVM (Kecman, 2001) 


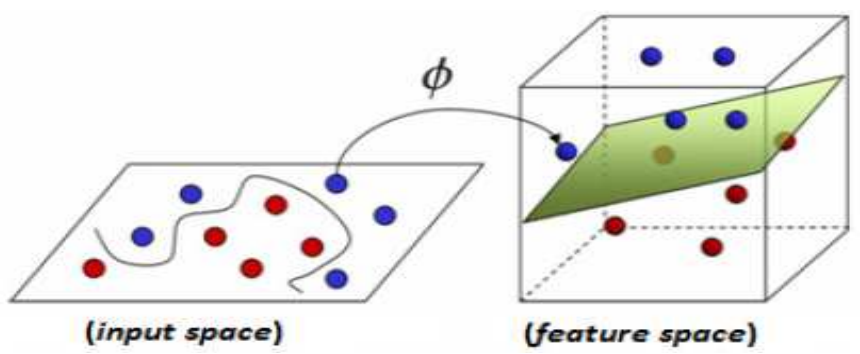

Fig-2: Mapping in multidimensional space

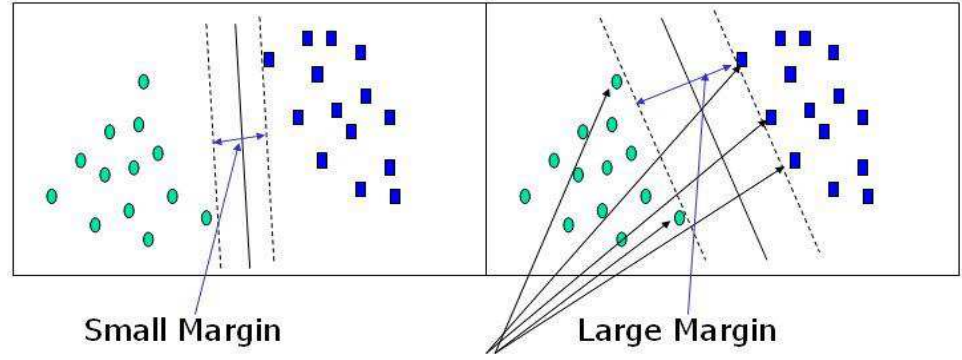

Support Vectors

Fig -3: Margins and support vectors

As we add additional predictor variables (attributes), the data points can be represented in $N$-dimensional space, and a $(N-1)$ dimensional hyperplane can separate them.

The goal of SVM modeling is to find the optimal hyperplane that separates clusters of vectors in such a way that cases with one category of the target variable are on one side of the plane and cases with the other category are on the other size of the plane. The vectors near the hyperplane are the support vectors. The concept of a kernel mapping function is very powerful. It allows SVM models to perform separations even with very complex boundaries such as shown on Fig.4 [24] .

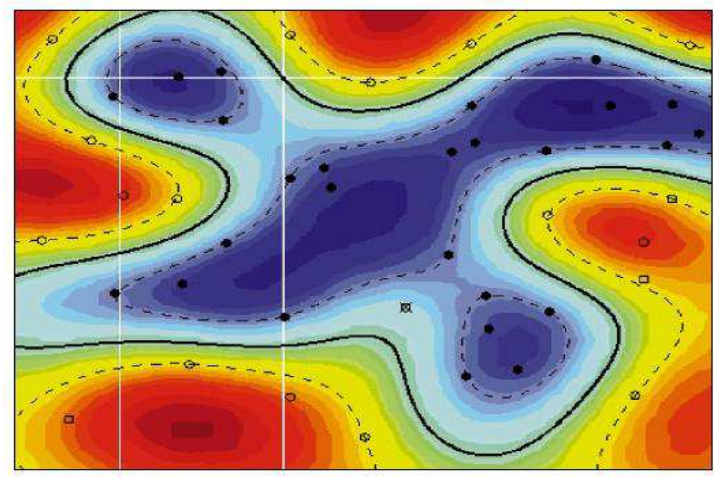

Fig - 4: Separation with kernel mapping

\subsection{Regression using SVM}

Now we shall define the basic problem of training for regression problems.

The model of machine learning has $\boldsymbol{l}$ data for training with which it tries to establish ("learn") relationship between input and output, expressed by some function. The set of data for training $\mathrm{D}$ is given by

$$
D=\left\{\left(x_{i}, y_{i}\right) \in R^{n} x R, i=1,2, \ldots . . l\right\}
$$

This set consists of the pairs $\left(\mathbf{x}_{1}, \mathbf{y}_{1}\right),\left(\mathbf{x}_{2}, \mathbf{y}_{2}\right), \ldots,\left(\mathbf{x}_{l}, \mathbf{y}_{l}\right)$, where inputs $\mathbf{x}$ are $\mathbf{n}$-dimensional vectors $\boldsymbol{x}_{i} \in \boldsymbol{R}^{n}$, and the responses of the model (outputs) $\boldsymbol{y}_{\boldsymbol{i}} \in \boldsymbol{R}$ has continual values. SVM as approximation function uses some function $\mathbf{f}(\mathbf{x}, \mathbf{w})$, where $\mathbf{w}$ are the subject of training $\square$ In $\varepsilon-S V$ regression, the goal is to find a function $f$ that has at most $\varepsilon$ deviation from the actually obtained targets $\mathbf{y}_{\mathbf{i}}$ for all the training data and at the same time as flat as possible [19].

This function presents model of non-linear regression, because the resulting hiperplane is non-linear function over the ndimensional $\mathbf{x}$ - space.

Estimation of the regression model with SVM is based on estimation of the error of the approximation. There are several 
types of functions of errors (error or loss function). The most often used is square error $(\mathbf{y}-\mathbf{f})^{\mathbf{2}}$ and absolute error $|\boldsymbol{y}-\boldsymbol{f}|$. Another type of error is the so called Vapnik's general type of error, i.e. linear loss function with $\mathcal{E}$ insensitive zone, defined as:

$$
|y-f(x, w)|_{\varepsilon}= \begin{cases}0 & \text { if }|y-f(x, w)| \leq \varepsilon \\ |y-f(x, w)|-\varepsilon & \text { if }|y-f(x, w)|>\varepsilon\end{cases}
$$

We can see that the error is zero if difference between real value $\mathbf{y}$ and obtained (predicted) value $\mathbf{f}(\mathbf{x}, \mathbf{w})$ is smaller than $\mathcal{E}$. Vapnik's function of error with $\mathcal{E}$ insensitivity zone defines tube, as it is presented on figure 5 . If the predicted value is in the tube, the error is 0 . For all other predicted values, which are out of the tube, the error is equal to the difference between absolute value of difference between actual and predicted value, and the radius of the tube $\varepsilon$.

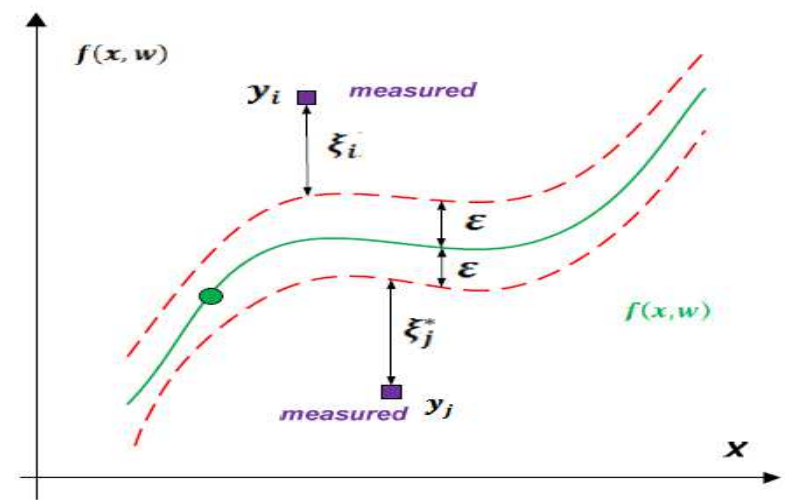

Fig-5: $\varepsilon$ - tube (Kecman, 2001)

Because of easier understanding of solving the regression problem using SVM (i.e. SVR), the simplest way to explain it is to take the problem of linear regression first. The function $\mathbf{f}(\mathbf{w}, \mathbf{x})$ will be linear:

$$
f(x, w)=w^{T} x+b
$$

We shall now present procedure of solving linear regression using SVMs.

In fact, in this case we want to find function $\mathbf{f}(\mathbf{x}, \mathbf{w})$ given with (4) , such that it approximates all pairs $\left(\mathbf{x}_{\mathbf{i}}, \mathbf{y}_{\mathbf{i}}\right)$ with $\mathcal{E}$ precision. Fig. 6 shows that situation. Because the width of the tube is $\frac{2 \varepsilon}{\|\boldsymbol{w}\|}$, the maximal permitted deviation of pairs $\left(\mathbf{x}_{\mathbf{i}}, \mathbf{y}_{\mathbf{i}}\right)$ in this tube will be obtained by minimizing $\|\boldsymbol{w}\|$.

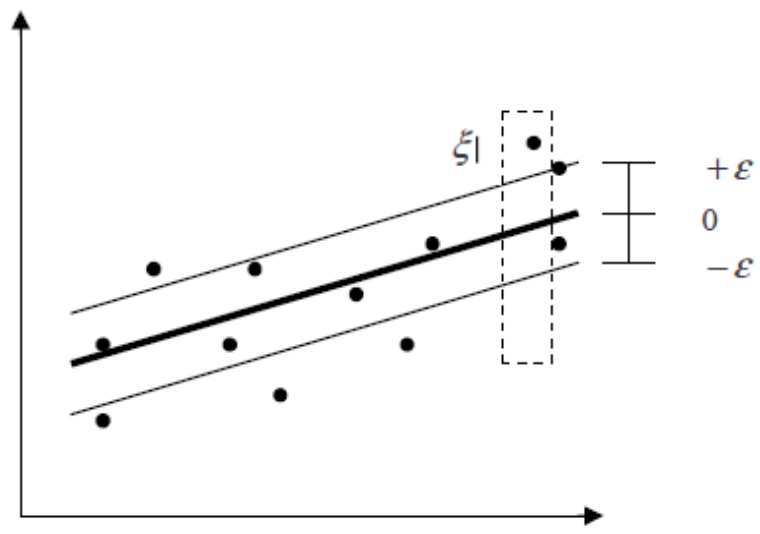

Fig-6: $\varepsilon$ - tube when the approximation function is linear

The optimization problem presented above is difficult to solve because it depends on $\|\boldsymbol{w}\|$, the norm of $\mathbf{w}$, which involves a square root. Fortunately it is possible to alter the equation by substituting $\|\boldsymbol{w}\|$ with $\frac{1}{2}\|\boldsymbol{w}\|^{2}$ (the factor of $1 / 2$ being used for mathematical convenience) without changing the solution (the minimum of the original and the modified equation have the same $\mathbf{w}$ and $\mathbf{b}$ ). This is a convex optimization problem. More clearly:

$$
\operatorname{minimize} \frac{1}{2}\|\boldsymbol{w}\|^{2}
$$

$$
\text { subject to }\left\{\begin{array}{l}
\boldsymbol{y}_{i}-\boldsymbol{w} \boldsymbol{x}_{i}-\boldsymbol{b} \leq \varepsilon \\
\boldsymbol{w} \boldsymbol{x}_{i}+b-\boldsymbol{y}_{i} \leq \varepsilon
\end{array}\right.
$$

Because SVM are developed in the frame of SRM (structurel risk minimization) which tends to minimize expected risk $\mathrm{R}$, according to Vapnik, the term for empirical risk is defined first:

$$
\boldsymbol{R}_{\text {emp }}^{\varepsilon}(w, b)=\frac{1}{l} \sum_{i=1}^{l}\left|y_{i}-w^{T} x_{i}-b\right|_{\varepsilon}
$$

$\boldsymbol{l}$ is the number of data for training. The main aim in SV algorithm is to minimize $\boldsymbol{R}_{\text {emp }}^{\varepsilon}$ and $\|\boldsymbol{w}\|$. We shall obtain the 
regression linear hyperplane $f(x, w)=w^{T} x+b \quad$ by minimizing of the real error:

$$
\boldsymbol{R}=\frac{1}{2}\|w\|^{2}+C\left(\sum_{i=1}^{l}\left|y_{i}-f\left(x_{i}, w\right)\right|_{\varepsilon}\right)
$$

The above convex optimization problem is feasible in cases where $f$ actually exists and approximates all pairs $\left(\boldsymbol{x}_{\boldsymbol{i}}, \boldsymbol{y}_{\boldsymbol{i}}\right)$ with $\varepsilon$ precision. Sometimes, some errors are allowed. Introducing slack variables $\xi_{\mathrm{i}}, \xi_{\mathrm{i}}^{*}$ (we can see them also on Fig. 5 when $\mathrm{f}$ is nonlinear) to cope with otherwise infeasible constraints of the optimization problem (5), the formulation becomes:

$$
\begin{gathered}
\xi_{i}=\left|y_{i}-f\left(x_{i}, w\right)\right|-\varepsilon \\
\xi^{*}{ }_{i}=\left|y_{i}-f\left(x_{i}, w\right)\right|-\varepsilon
\end{gathered}
$$

Now we can write the term (7) in the following w ay:

$$
\boldsymbol{R}_{w, \xi, \xi_{i}^{*}}=\left[\frac{1}{2}\|\boldsymbol{x}\|^{2}+\boldsymbol{C}\left(\sum_{i=1}^{l} \xi_{i}+\sum_{i=1}^{l} \xi_{i}^{*}\right)\right]
$$

With the following constraints:

$$
\begin{gathered}
\mathbf{y}_{\mathbf{i}}-\mathbf{w}^{\mathbf{T}} \mathbf{x}_{\mathbf{i}}-\mathbf{b} \leq \varepsilon+\xi_{i} \quad i=1, \ldots ., l \\
\mathbf{w}^{\mathbf{T}} \mathbf{x}_{\mathbf{i}}+\mathbf{b}-\mathbf{y}_{\mathbf{i}} \leq \varepsilon+\xi_{i}^{*} \quad i=1, \ldots . ., l \\
\xi_{i} \geq 0 \quad i=1, \ldots, l \\
\xi_{i}^{*} \geq 0 \quad i=1, \ldots, l
\end{gathered}
$$

This optimization problem can be solved using formulation of Lagrange [19]:

$$
\begin{aligned}
& L\left(w, b, \xi_{i}, \xi_{i}^{*}, \alpha_{i}, \alpha_{i}^{*}, \beta_{i}, \beta_{i}^{*}\right)=\frac{1}{2} w^{T} w+ \\
& +C\left(\sum_{i=1}^{l} \xi_{i}+\sum_{i=1}^{l} \xi_{i}^{*}\right)-\sum_{i=1}^{l} \alpha_{i}^{*}\left[y_{i}-w^{T} x_{i}-b+\varepsilon+\xi_{i}^{*}\right]- \\
& -\sum_{i=1}^{l} \alpha_{i}\left[\boldsymbol{w}^{T} \boldsymbol{x}_{i}+\boldsymbol{b}-\boldsymbol{y}_{i}+\varepsilon+\xi_{i}\right]- \\
& -\sum_{i=1}^{l}\left(\beta_{i} \xi_{i}+\beta_{i}^{*} \xi_{i}^{*}\right)
\end{aligned}
$$

After solving the dual regression problem, the following parameters which defined optimal regression hyper plane $\mathbf{f}(\mathbf{x}, \mathbf{w})$ is obtained: optimal weigh vector $w_{0}$ :

$$
\boldsymbol{w}_{0}=\sum_{i=1}^{l}\left(\alpha_{i}^{*}-\alpha_{i}\right) \boldsymbol{x}_{i}
$$

And optimal $\mathbf{b}_{0}$ :

$$
b_{0}=\frac{1}{l} \sum_{i=1}^{l}\left(y_{i}-x_{i}^{T} w_{0}\right)
$$

The best regression hyper plane is defined by the term:

$$
z=f(x, w)=w_{0}^{T} x+b_{0} .
$$

But, the most of the regression problems does not have linear nature. Solving the problem with non-linear regression using SVMs is accomplished by considering the linear regression hyper plane in the new feature space.

The basic idea of forming SVMs for creating a function of nonlinear regression is to map the input vectors $\boldsymbol{x} \in \boldsymbol{R}^{\boldsymbol{n}}$ from the input space to vectors $\mathbf{z}$ from multi dimensional future space $\mathbf{F}(\mathbf{z}=\boldsymbol{\Phi}(\mathbf{x}))$, where $\boldsymbol{\Phi}$ is mapping function from $\boldsymbol{R}^{\boldsymbol{n}}$ to $\boldsymbol{R}^{f}$ and to solve the linear regression problem in the new feature space. The function $\boldsymbol{\Phi}(\mathbf{x})$ is fixed and given in advance and is called kernel function. The input, $\mathbf{x}$ - space is defined by the input vectors $\mathbf{x}_{\mathbf{i}}$, while the future space $\mathbf{F}$ ( $\mathbf{z}$ space) is defined by the components of the $\mathrm{z}$ vector. Applying the SVM formulation, this way of mapping allows obtaining linear regression hyper plain using training algorithm in $\mathrm{z}$ - space. It is expected that using this approach will lead to solving the problem of quadratic optimization with limitations given by inequalities in $\mathbf{z}$ - space.

Many kernel mapping functions can be used - probably an infinite number. But a few kernel functions have been found to work well for a wide variety of applications, for example: linear function, polynomial function, radial basis function and sigmoid function. The default and recommended kernel function is the radial basis function (RBF).

There are few parameters which can be used for training during the modeling of SVM for solving regression problems. The most significant are insensitivity zone $\mathcal{E}$ and penalty parameter $\mathbf{C}$. The both parameters are chosen by the user.

\subsection{Predicting Real Time of Construction with SVM}

\section{Model}

In this paper, for prediction of the real time of construction time using SVM model is used the software package DTREG 
[25]. DTREG offers several types of SVM models, for classification and for regression tasks.

DTREG provides four kernel functions, linear, polynomial, radial basis function ( $R B F$ ) and sigmoid ( $\mathrm{S}$-shaped). There is no way in advance to know which kernel function will be best for an application, but the RBF function $\left(\boldsymbol{\Phi}\left(\mathbf{x}_{\mathbf{i}}, \mathbf{x}_{\mathbf{j}}\right)=\right.$

$=\exp \left(-\right.$ gamma $\left.^{*}\left|\mathbf{x}_{\mathbf{i}}-\mathbf{x}_{\mathbf{j}}\right|^{2}\right)$ has been found to do best job in the majority of cases. RBF is the default and recommended kernel function. The RBF kernel non-linearly maps samples into a higher dimensional space, so it can handle nonlinear relationships between target categories and predictor attributes; a linear basis function cannot do this. Furthermore, the linear kernel is a special case of the RBF.

There is a tolerance factor that controls when DTREG stops the iterative optimization process. The default value usually works well, but the tolerance can be reduced to generate a more accurate model or increase the value to reduce the computation time. This parameter is called the Epsilon value in some implementations of SVM.

DTREG can generate SVM model giving also a report on the relative significance of predictor variables.

For validating a SVM model in this paper, DTREG uses Vfold cross validation method. This means that $V$ SVM models will be constructed with $(V-1) / V$ proportion of the rows being used in each model. The remaining rows are then used to measure the accuracy of the model. The final model is built using all data rows. This method has the advantage of using all data rows in the final model, but the validation is performed in separately constructed models.

The accuracy of an SVM model is largely dependent on the selection of the model parameters such as C, Gamma, etc. DTREG provides two methods for finding optimal parameter values.

When DTREG uses SVM for regression problems, criterion for minimizing total error is used to find optimal parameters for determining optimal function value.

The SVM implementation used by DTREG is partially based on the outstanding LIBSVM project by Chih-Chung Chang and Chih-Jen Lin [6]. They have made both theoretical and practical contributions to the development of support vector machines.

The results of the implementation of SVM model using package DTREG for predicting the real time of construction is given bellow on Table 4 to Table 6 . For the accuracy of the model it is very important how we choose the target and predictor variables. Considering the Eq. (2), for this model, as target variable is chosen $\ln$ (real time) and for predicted variables are chosen: $\ln ($ contracted time), $\ln$ (real price) and $\ln$ (contracted price). 
Table-4: SVM model for predicting real time of construction

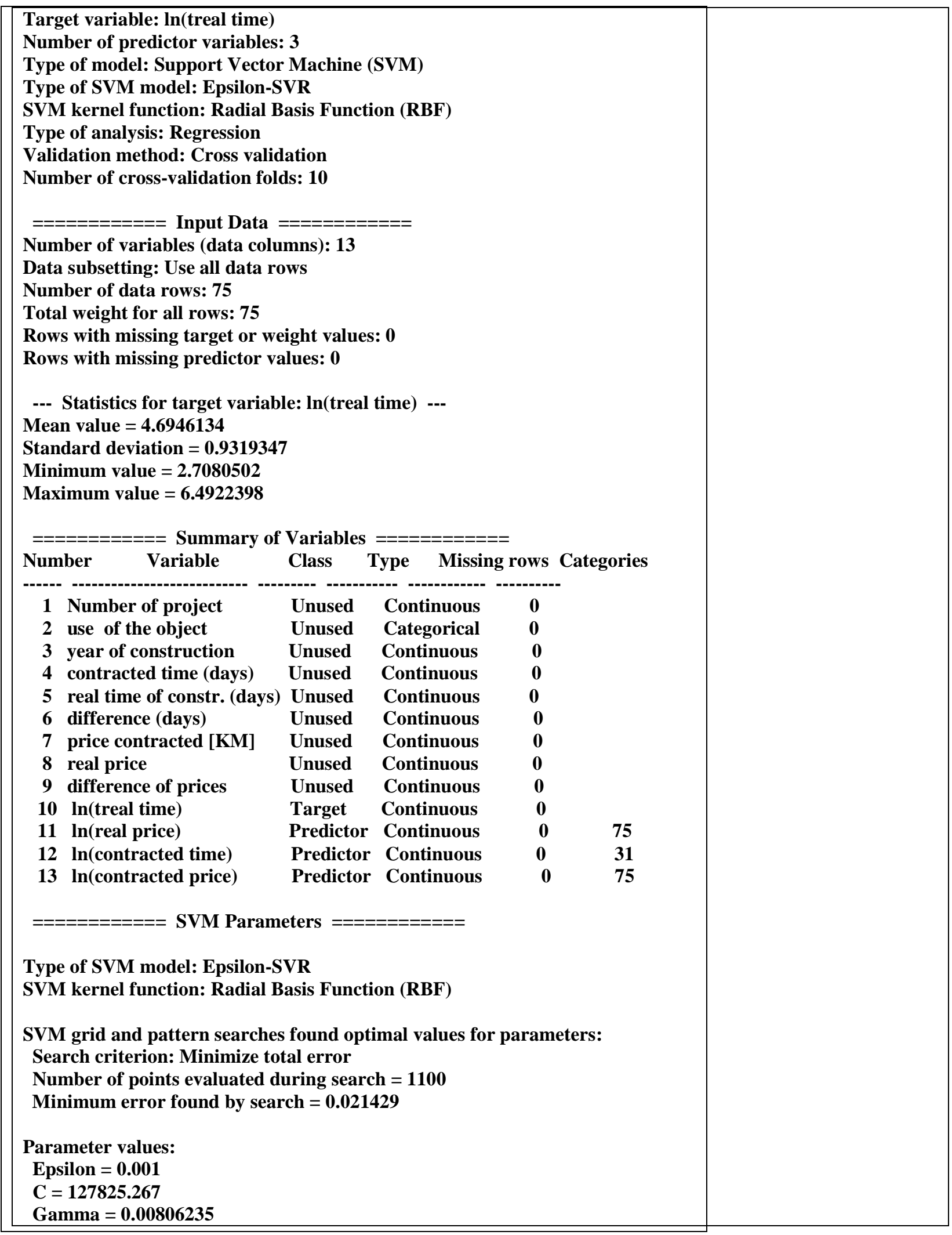




\section{$P=0.04609048$}

Number of support vectors used by the model $=\mathbf{5 0}$

Table -5: Statistics for training data of the model

\section{--- Training Data ---}

Mean target value for input data $=4.6946134$

Mean target value for predicted values $=4.6646417$

Variance in input data $=0.8685023$

Residual (unexplained) variance after model fit $=0.0205836$

Proportion of variance explained by model $\left(\mathrm{R}^{\wedge} 2\right)=0.97630$ $(97.630 \%)$

Coefficient of variation $(\mathrm{CV})=0.030560$

Normalized mean square error $(\mathrm{NMSE})=0.023700$

Correlation between actual and predicted $=0.988682$

Maximum error $=0.6236644$

RMSE (Root Mean Squared Error) $=0.1434697$

MSE (Mean Squared Error) $=0.0205836$

MAE (Mean Absolute Error) $=0.0923678$

MAPE (Mean Absolute Percentage Error) = 2.0150009

Table -6: Statistics for validation data of the model

\section{--- Validation Data ---}

Mean target value for input data $=4.6946134$

Mean target value for predicted values $=4.6650366$

Variance in input data $=0.8685023$

Residual (unexplained) variance after model fit $=$ 0.0225357

Proportion of variance explained by model $\left(\mathrm{R}^{\wedge} 2\right)=$ $0.97405(97.405 \%)$

Coefficient of variation $(\mathrm{CV})=0.031977$

Normalized mean square error $(\mathrm{NMSE})=0.025948$

Correlation between actual and predicted $=0.987501$

Maximum error $=0.6219012$

RMSE (Root Mean Squared Error) $=0.1501191$

MSE (Mean Squared Error) $=0.0225357$

MAE (Mean Absolute Error) $=0.0993009$

MAPE (Mean Absolute Percentage Error) $=2.1722942$ 
As we can see from the statistic for the validation data which are used for estimation of the accuracy of the model, $\mathbf{R}^{2}=$ $0.97405(\mathbf{9 7 . 4 0 5 \%})$, and MAPE $=\mathbf{2 . 1 7 2 2 9 4 2}$, which is a significant improvement over the linear model.

\section{CONCLUSIONS}

Prediction of construction time using SVM (Support Vector Machine) model is presented in this paper. The analysis covered 75 objects structured in the period of 1999 to 2011 in the federation of Bosnia and Herzegovina. First, conventional linear regression model has been applied to the data using the well known "time cost" model, and after that, the predictive model with SVM was build and applied to the same data. The results show that the predicting with SVM was significantly more accurate.

A Support Vector Machine is a relatively new modeling method that has shown great promise at generating accurate models for a variety of problems.

The authors hope that the presented model encourages serious discussions about the topic presented here and that they will prove as useful for improving planning in the construction industry in general.

\section{REFERENCES}

[1] D. Basak, S. Pal and D. C. Patranabis: Support Vector Regression, Neural Information Processing, Vol. 11, No.10, oct. 2007

[2] B. E. Boser, I. M. Guyon, and V. N. Vapnik. A training algorithm for optimal margin classifiers. In D. Haussler, editor, Proceedings of the Annual Conference on Computational Learning Theory, pages 144-152, Pittsburgh, PA, July 1992. ACM Press.

[3] Bromilow F. J.: Contract time performance expectations and the reality. Building Forum, 1969, Vol.1(3): pp. 70-80.

[4] Car - Pušić D.. Methodology of anticipating sustainable construction time, (in croatian) PhD thesis, 2004, Faculty of Civil Engineering, University of Zagreb.

[5] D.W.M. Chan. and M.M.A. Kumaraswamy.: Study of the factors affecting construction duration in Hong Kong, Construction management and economics, 13(4): pp. 319333, 1995

[6]Chang, Chih-Chung and Chih-Jen Lin. LIBSVM - A Library for Support Vector Machines. April, 2005. http://www.csie.ntu.edu.tw/ cjlin/libsvm/

[7] V. Cherkassky and F. Mulier. Learning from Data. John Wiley and Sons, New York, 1998.

[8] Choudhry I. and Rajan S.S.. Time - cost relationiship for residential construction in Texas. College Station, 2003, Texas A\&M University.

[9] C. Cortes and V. Vapnik. Support vector networks. Machine Learning, 20:273-297, 1995.
[10] H. Drucker, C. J. C. Burges, L. Kaufman, A. Smola, and V. Vapnik. Support vector regression machines. In M. C. Mozer, M. I. Jordan, and T. Petsche, editors, Advances in Neural Information Processing Systems 9, pages 155-161, Cambridge, MA, 1997. MIT Press.

[11] Guyon, B. Boser, and V. Vapnik. Automatic capacity tuning of very large VC-dimension classifiers. In S. J. Hanson, J. D. Cowan, and C. L. Giles, editors, Advances in Neural Information Processing Systems 5, pages 147-155. Morgan Kaufmann Publishers, 1993.

[12] S. Haykin. Neural Networks : A Comprehensive Foundation. Macmillan, New York, 1998. 2nd edition.

[13] S. Haykin,: Neural Networks: A Comprehesive Foundation, 2nd ed., Pearson Education Inc., Canada, 2005

[ 14] M. A. Hearst, B. Sch“olkopf, S. Dumais, E. Osuna, and J. Platt. Trends and controversies-support vector machines. IEEE Intelligent Systems, 13:18-28, 1998.

[15] Kaka A. and Price A.D.F.: Realitionship between value and duration of contruction projects, Construction Management and Economics, 1991, Vol. 9: pp. 383-400.

[16] V. Kecman, Learning and Soft Computing, Support Vector Machines, Neural Networks and Fuzzy Logic Models, Massachusetts Institute of Technology (MIT), USA, 2001

[17] D. Mattera and S. Haykin. Support vector machines for dynamic reconstruction of a chaotic system. In B. Sch"olkopf, C. J. C. Burges, and A. J. Smola, editors, Advances in Kernel Methods-Support Vector Learning, pages 211-242, Cambridge, MA, 1999. MIT Press.

[18] K.-R. M“ uller, A. Smola, G. R“atsch, B. Sch“olkopf, J. Kohlmorgen, and V. Vapnik. Predicting time series with support vector machines. In W. Gerstner, A. Germond, M. Hasler, and J.-D. Nicoud, editors, Artificial Neural Networks ICANN'97, pages 999-1004, Berlin, 1997. Springer Lecture Notes in Computer Science, Vol. 1327.

[19] I. Pesko: Model for estimation of costs and time of construction for urban traffic arteries, $\mathrm{PhD}$ Thesis (in serbian), Novi Sad, 2013

[20] B. Sch"olkopf, C. Burges, and V. Vapnik. Extracting support data for a given task. In U. M. Fayyad and R. Uthurusamy, editors, Proceedings, First International Conference on Knowledge Discovery \& Data Mining, Menlo Park, 1995. AAAI Press

[21] B. Sch“olkopf, C. Burges, and V. Vapnik. Incorporating invariances in support vector learning machines. In $\mathrm{C}$. von der Malsburg,W. von Seelen, J. C. Vorbr '. uggen, and B. Sendhoff, editors, Artificial Neural Networks ICANN'96, pages 47-52, Berlin, 1996. Springer Lecture Notes in Computer Science, Vol. 1112.

[22] B. Sch“olkopf, C. J. C. Burges, and A. J. Smola, editors. Advances in Kernel Methods-Support Vector Learning. MIT Press, Cambridge, MA, 1999a.

[23] B. Sch“olkopf and A. J. Smola. Learning with Kernels. MIT Press, 2002.

[24] P. Sherrod: DTREG Predictive Modeling Software tutorial, 2013, www.dtreg.com 
[25] P. Sherrod: DTREG Predictive Modeling Software, 2013, www.dtreg.com

[26] A.J. Smola, B. Sch“olkopf: A tutorial on Support Vector Regression, sept, 2003

[27] M. Stitson, A. Gammerman, V. Vapnik, V. Vovk, C. Watkins, and J.Weston. Support vector regression with ANOVA decomposition kernels. In B. Sch“olkopf, C. J. C. Burges, and A. J. Smola, editors, Advances in Kernel Methods-Support Vector Learning, pages 285-292, Cambridge, MA, 1999. MIT Press.

[28] V. Vapnik and A. Lerner: Pattern recognition using generalized portrait method. Automation and Remote Control, 24: 774-780, 1963.

[ 29] V. Vapnik and A. Chervonenkis. A note on one class of perceptrons. Automation and Remote Control, 25, 1964.

[30] V. Vapnik and A. Chervonenkis. Theory of Pattern Recognition [in Russian]. Nauka, Moscow, 1974. (German Translation:W. Wapnik \& A. Tscherwonenkis, Theorie der Zeichenerkennung,Akademie-Verlag, Berlin, 1979).

[31] V. N. Vapnik. Estimation of Dependences Based on Empirical Data. Springer, Berlin, 1982.

[32] V. Vapnik. The Nature of Statistical Learning Theory. Springer, New York, 1995.

[33] V. Vapnik, S. Golowich, and A. Smola. Support vector method for function approximation, regression estimation, and signal processing. In M. C. Mozer,M. I. Jordan, and T. Petsche, editors, Advances in Neural Information Processing Systems 9, pages 281-287, Cambridge, MA, 1997. MIT Press. [34] www.http://en.wikipedia.org/wiki/Regression_analysis

[35] www.http://en.wikipedia.org/wiki/Linear_regression

[36]www.http://en.wikipedia.org/wiki/Coefficient

_of_determination

[37]www.http://en.wikipedia.org/wiki/Mean_absolute

_percentage_error

[38] V. Zujo: Contribution of Construction Time Planning to Construction Project Management (in bosnian). PhD thessis, 2008, Faculty of Civil Engineering, University „Džemal Bijedić" of Mostar.

[39] V. Zujo, V. Zileska-Pancovska, M.Chechez; Prediction model for sustainable construction project duration, 15th International Symposium (of MASE), Struga, Macedonia, 1821 Sept., 2013

\section{BIOGRAPHIES}

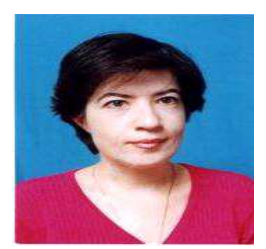

Silvana Petruseva, Phd (informatics, Msc (mathematics , application in arti-ficial intelligence), Bsc ( mathematicsinformatics). Assist. prof. on Faculty of Civil Engineering (Department of mathematics), at the University "Ss. Cyril and Methodius" in Skopje, FYR Mace-donia. Phd thesis was from the area of artificial intelligence (neural networks, agent learning), Area of interest and investigation is development and application of neural networks. She has published in the country and abroad.

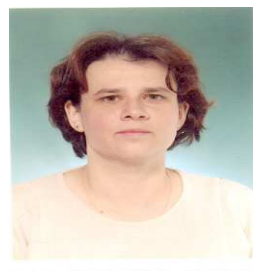

Valentina Zileska-Pancovska, PhD, Msc and Bsc (in Civil Engineering), Full professor at the Faculty of Civil Engineering (department of technology and management of construction, currently head of the department), at University "Ss. Cyril and Methodius" in Skopje, FYR of Macedonia. Before becoming an academic, she worked for a construction company. She has published in the Macedonia and abroad. Area of research is organization, technology and menagement in construction, especially construction project management.

Vahida Zujo, PhD, Msc and Bsc (Civil Engineering), Professor at the Faculty of Civil Engineering,, currently Vice rector of the University "Dzemal Bjedic" in Mostar, Bosnia and Herzegovina. Before becoming an academic, she worked for a construction company. She has published in the country and abroad. Area of research is organization, technology and menagement in construction 